\title{
COHOMOLOGY IN TENSORED CATEGORIES
}

\author{
MICHAEL BARR
}

\section{Introduction}

Both Mac Lane 1965, Beck 1967] have recently defined cohomology theories for algebras in abstract categorial setting. Mac Lane's theory is an abstract formaization of the (normalised) bar construction (see $[$ Mac Lane 1963, p. 144] for example). Since, however, his proof of the normalization theorem (p. 236) remains perfectly valid, the normalized bar construction can be replaced by the un-normalized one. It is the purpose of this paper to show that under reasonable conditions Beck's cohomology is naturally equivalent to a slight modification of Mac Lane's. All notation not explicitly defined is taken from Mac Lane 1963.

The proof given here is mainly just a modification of that of [Barr \& Beck 1966]. However sections 2, 3, and 4 are devoted to showing that the techniques used there can all be applied to the general categorical situation.

\section{Algebras}

If $\mathcal{D}$ is a tensored category, $\operatorname{Alg}(\mathcal{D})$ denotes the category of $\mathcal{D}$-algebras as described in Mac Lane 1963, p. 79]. The purpose of this section is to show:

2.1. TheOrem. Suppose $\mathcal{D}$ has countable coproducts which commute with the $\otimes$. Then there is a triple $\mathbf{T}=\left(T, \eta\right.$, mu) on $\mathcal{D}$ such that $\operatorname{Alg}(\mathcal{D}) \cong \mathcal{D}^{\mathbf{T}}$.

ProOF. We will first show that the underlying functor $U: \operatorname{Alg}(\mathcal{D}) \longrightarrow \mathcal{D}$ has a left adjoint $F$. Then if $\eta: 1 \longrightarrow U F$ and $\epsilon: F U \longrightarrow 1$ and the adjointness morphisms, it is known that that $\mathbf{T}=(U F, \eta, U \epsilon F)$ is a triple [Barr \& Beck 1966, Beck 1967]. Then we complete the proof by exhibiting a natural equivalence $\operatorname{Alg}(\mathcal{D}) \cong \mathcal{D}^{\mathbf{\top}}$.

2.2. Definition. Let $A_{1}, \ldots, A_{n}$ be objects of $\mathcal{D}$. We define $A_{1} \otimes \cdots \otimes A_{n}$ inductively to be $K$ if $n=0$ and $\left(A_{1} \otimes \cdots \otimes A_{n_{1}}\right) \otimes A_{n}$ for $n>0$. If each $A_{i}=A$, we will also denote this by $A^{(n)}$. It follows from the defintion of a tensored category that there is a unique ismorphism which we denote by $\sigma(m, n): A^{(m)} \otimes A^{(n)} \longrightarrow A^{(m+n)}$.

We now let $F(A)=\sum_{n \geq 0} A^{(n)}$. To describe an algebra structure on $F(A)$, let $\alpha_{n}$ : $A^{(n)} \longrightarrow F(A)$ be the inclusion. Since tensor commutes with this coproduct, a map $\pi_{A}$ : $F(A) \otimes F(A) \cong \sum_{n, m \geq 0} A^{(n)} \otimes A^{(m)} \longrightarrow F(A)$ is determined by requiring that $\pi\left(\alpha_{n} \otimes \alpha_{m}\right)=$ $\alpha_{n+m} \sigma(n, m)$. Then I claim that $F(A), \pi, \alpha_{0}$ (as unit) form an object of $\operatorname{Alg}(\mathcal{D})$. We must

(c) Michael Barr, 1966. Permission to copy for private use granted. 
show three identities [Mac Lane 1965, p. 79]. The first is that if $e_{F(A)}: K \otimes F(A) \longrightarrow F(A)$ is the Mac Lane isomorphism, then $\pi\left(\alpha_{0} \otimes 1\right)=e_{F(A)}$. But $\sigma(0, n)=e_{A}(n)$, and, by the naturality of $e$ we have

$$
\pi\left(\alpha_{0} \otimes 1\right)\left(1 \otimes \alpha_{m}\right)=\pi\left(\alpha_{0} \otimes \alpha_{m}\right)=\alpha_{m} \sigma(0, m)=e_{F(A)}\left(1 \otimes \alpha_{m}\right)
$$

Since the $1 \otimes \alpha_{m}$ are inclusions to a direct sum, this gives the result. The second is derived similarly. The third is that

$$
\pi(1 \otimes \pi)=\pi(\pi \otimes 1): F(A) \otimes F(A) \otimes F(A) \longrightarrow F(A)
$$

But $F(A) \otimes F(A) \otimes F(A)$ is a direct sum

$$
\sum_{m, n, p \geq 0} A^{(m)} A^{(n)} \otimes A^{(p)}
$$

We have

$$
\begin{aligned}
\pi(1 \otimes \pi)\left(\alpha_{m} \otimes \alpha_{n} \otimes \alpha_{p}\right) & =\pi\left(\alpha_{m} \otimes \pi\left(\alpha_{n} \alpha_{p}\right)\right) \\
& =\pi\left(\alpha_{m} \otimes \alpha_{n+p} \sigma(n, p)\right)=\pi\left(\alpha_{m} \otimes \alpha_{n+p}\right)(1 \otimes \sigma(n, p)) \\
& =\alpha_{m+n+p} \sigma(m, n+p)(1 \otimes \sigma(n, p))
\end{aligned}
$$

But

$$
\sigma(m, n+p)(1 \otimes \sigma(n, p)): A^{(m)} \otimes A(n) \otimes A^{(p)} \longrightarrow A^{(m+n+p)}
$$

is a Mac Lane isomorphism, which by uniqueness must equal

$$
\sigma(m+n, p)(\sigma(m, n) \otimes 1)
$$

Then we get

$$
\begin{gathered}
\alpha_{m+n+p} \sigma(m+n, p)(\sigma(m, n) \otimes 1)=\pi\left(\alpha_{m+n} \otimes \alpha_{p}\right)(\sigma(m, n) \otimes 1)=\pi\left(\alpha_{m+n} \sigma(m, n) \otimes \alpha_{p}\right) \\
=\pi\left(\pi\left(\alpha_{m} \otimes \alpha_{n}\right) \otimes \alpha_{p}\right)=\pi(\pi \otimes 1)\left(a l p h a_{m} \otimes \alpha_{n} \otimes \alpha_{p}\right)
\end{gathered}
$$

Since the $\alpha_{n} \otimes \alpha_{n} \otimes \alpha_{p}$ are a direct family of inclusions, the result follows.

Now suppose $\left(\Lambda, p_{\Lambda}, u_{\Lambda}\right)$ is in $\operatorname{Alg}(\mathcal{D})$ and $f: A \rightarrow \Lambda$ is in $\mathcal{D}$. We prove adjointness by showing that there is a unique map $f^{*}: F(A) \longrightarrow \Lambda$ in $\operatorname{Alg}(\mathcal{D})$ such that $f^{*} \alpha_{1}=f$. (At least this proves adjointness with $\alpha_{1}: F A \longrightarrow U F(A)$ as one of the adjointness morphisms cf. Mac Lane 1965, p. 3].) We define $f^{*}$ by requiring that $f^{*} \alpha_{0}=u_{\Lambda}, f^{*} \alpha_{1}=f$, and $f^{*} \alpha_{i}=p_{\Lambda}\left(f^{*} \alpha_{i-1} \otimes f\right)$ for $i>1$. To show this in $\operatorname{Alg}(\mathcal{D})$ we must show that it preserves the unit which follows from the definition of $f^{*} \alpha_{0}$ and that $f^{*} \pi=p_{\Lambda}\left(f^{*} \otimes f^{*}\right)$. By 
induction on $m+n$,

$$
\begin{aligned}
f^{*} \pi\left(\alpha_{m} \otimes \alpha_{n}\right) & =f^{*} \alpha_{m+n} \sigma(m, n)=p_{\Lambda}\left(f^{*} \alpha_{n+m-1} \otimes f\right) \sigma(m, n) \\
& =p_{\Lambda}\left(f^{*} \alpha_{n+m-1} \otimes f\right)(\sigma(m, n-1) \otimes 1)=p_{\Lambda}\left(f^{*} \alpha_{m+n-1} \sigma(m, n-1) \otimes f\right) \\
& =p_{\Lambda}\left(f^{*} \pi\left(\alpha_{m} \otimes \alpha_{n-1}\right) \otimes f\right)=p_{\Lambda}\left(p_{\Lambda}\left(f^{*} \otimes f^{*}\right)\left(\alpha_{m} \alpha_{n-1}\right) \otimes f\right) \\
& =p_{\Lambda}\left(p_{\Lambda}\left(f^{*} \alpha_{m} \otimes f^{*} \alpha_{n-1}\right) \otimes f\right)=p_{\Lambda}\left(p_{\Lambda} \otimes 1\right)\left(f^{*} a l p h a_{m} \otimes f^{*} \alpha_{n-1} \otimes f\right) \\
& =p_{\Lambda}\left(1 \otimes p_{\Lambda}\right)\left(f^{*} \alpha_{m} \otimes f^{*} \alpha_{n-1} \otimes f\right)=p_{\Lambda}\left(f^{*} \alpha_{m} \otimes p_{\Lambda}\left(f^{*} \alpha_{n-1} f\right)\right) \\
& =p_{\Lambda}\left(f^{*} \alpha_{m} \otimes f^{*} \alpha_{n}\right)=p_{\Lambda}\left(f^{*} \otimes f^{*}\right)\left(\alpha_{m} \otimes \alpha_{n}\right)
\end{aligned}
$$

Again, this implies that $f^{*} \pi=p_{\Lambda}\left(f^{*} \otimes f^{*}\right)$. Suppose $g$ is another map with $g \alpha_{1}=f$. Then $g \alpha_{0}=u_{\Lambda}=f^{*} \alpha_{0}$ by the unitary condition, $f \alpha_{1}=f=f^{*} \alpha_{1}$ by assumption, and if we assume $g \alpha_{i-1}=f^{*} \alpha_{i-1}$, then

$$
\begin{aligned}
f^{*} \alpha_{i} & =p_{\Lambda}\left(f^{*} \alpha_{i-1} \otimes f\right)=p_{\Lambda}\left(g \alpha_{i-1} \otimes g \alpha_{1}\right)=p_{\Lambda}(g \otimes g)\left(\alpha_{i-1} \otimes \alpha_{1}\right) \\
& =g \pi\left(\alpha_{i-1} \otimes \alpha_{1}\right)=g \sigma(i-1,1) \alpha_{i}=g \alpha_{i}
\end{aligned}
$$

(since $\sigma(i-1,1)$ is the identity isomorphism.) Hence $f^{*} \alpha_{i}=g \alpha_{i}$ for each $i$. so $f^{*}=g$.

This shows adjointness, and therefore gives rise to a triple as noted about. Moreover, we have from [Barr \& Beck 1966] a natural functor $\Psi: \operatorname{Alg}(\mathcal{D}) \longrightarrow \mathcal{D}^{\boldsymbol{\top}}$ which takes $\left(\Lambda, p_{\Lambda}, u_{\Lambda}\right)$ to $(\Lambda, \lambda)$ where $\lambda: F(\Lambda) \longrightarrow \Lambda$ is the unique algebra map extending the identity map of $\Lambda$. Specifically, if $\beta_{i}: \Lambda^{(i)} \longrightarrow \Lambda$ are the direct system, then $\lambda \beta_{0}=u_{\Lambda}, \lambda \beta_{1}=1_{\Lambda}$ and $\lambda \beta_{i}=p_{\Lambda}\left(\lambda \beta_{i-1} \otimes 1_{\Lambda}\right)$. Notice that $\lambda \beta_{2}=p_{\lambda}$. This tells us how to construct an inverse functor. In particular, if $(X, \xi)$ is a $\mathbf{T}$-algebra we let $u_{X}=\xi \gamma_{0}$ and $p_{X}=\xi \gamma_{2}$ where $\gamma_{i}: X^{(i)} \longrightarrow F(X)$ are the inclusions. If these give $X$ the structure of a $\mathcal{D}$-algebra, then they clearly define a functor $\Phi$ which is a right inverse to $\Psi$. Let $\phi_{i}: F(X)^{(i)} \longrightarrow F(F(X))$ be the inclusion. Then we know that $\xi \gamma_{1}=1_{X}$ by definition of a $\mathbf{T}$-algebra. The other condition says that $\xi \cdot F \xi=\xi \cdot U \epsilon F(X)$. But $U \epsilon F(A): F(F(A)) \longrightarrow F(A)$ is just the map coming out of the algebra structure on $F(A)$. Hence $U \epsilon F(X) \cdot i_{2}=\pi_{X}$ : $F(X) \otimes F(X) \longrightarrow F(X)$. On the other hand, $F \xi \cdot \phi_{2}: F(X) \otimes F(X) \longrightarrow F(X)$ is just $\gamma_{2}(\xi \otimes \xi)$. Hence $\xi \gamma_{2}(\xi \otimes \xi)=\xi \pi_{X}: F(X) \otimes F(X) \longrightarrow X$.

$$
\begin{aligned}
\xi \gamma_{2}\left(1 \otimes \xi \gamma_{2}\right) & =\xi \gamma_{2}\left(\xi \gamma_{1} \otimes \xi \gamma_{2}\right)=\xi \gamma_{1}(\xi \otimes \xi)\left(\gamma_{1} \otimes \gamma_{2}\right) \\
& =\xi \pi_{X}\left(\gamma_{1} \otimes \gamma_{2}\right)=\xi \gamma_{2} \sigma(1,2)=\xi \gamma_{3} \sigma(2,1) \sigma(1,2) \\
& =\xi \pi_{X}\left(\gamma_{2} \otimes \gamma_{1}\right) \sigma(1,2)=\xi \gamma_{2}(\xi \otimes \xi)\left(\gamma_{2} \otimes g a m m a_{1}\right) \sigma(1,2) \\
& =\xi \gamma_{2}\left(\xi \gamma_{2} \otimes \xi \gamma_{1}\right) \sigma(1,2)=\xi \gamma_{2}\left(\xi \gamma_{2} \otimes 1\right) \sigma(1,2)
\end{aligned}
$$

which, strictly speaking, is the precise statement that $\xi \gamma_{2}$ is an associative law of composition. The unitary laws are left to the reader. Now we must show that $\Phi$ is a left inverse 
to $\Psi$ as well. To do this we start with $(X, \xi)$ in $\mathcal{D}^{\boldsymbol{\top}}$ and make it into an algebra using $\xi \gamma_{2}: X \otimes X \longrightarrow F(X) \longrightarrow X$ as the rule of composition and $\xi \gamma_{0}$ as unit. We now a new map $\zeta: F(X) \longrightarrow X$ by setting $\zeta \gamma_{0}=\xi \gamma_{0}, \zeta \gamma_{1}=1=\zeta \gamma_{1}$, and $\zeta \gamma_{i}=\xi \gamma_{2}\left(\zeta \gamma_{i-1} \otimes 1\right)$ for $i>1$. Assuming, however, that $\zeta \gamma_{i-1}=\xi \gamma_{i-1}$, we have

$$
\begin{aligned}
\zeta \gamma_{i} & =\xi \gamma_{2}\left(\zeta \gamma_{i-1}\right)=\xi \gamma_{2}\left(\xi \gamma_{i-1} \otimes 1\right)=\xi \gamma_{2}\left(\xi \gamma_{i-1} \otimes \xi \gamma_{1}\right) \\
& =\xi \gamma_{2}(\xi \otimes \xi)\left(\gamma_{i-1} \otimes \gamma_{1}\right)=\xi \pi_{X}\left(\gamma_{i-1} \otimes \gamma_{1}\right)=\xi \gamma_{i} \sigma(i-1.1)=\xi \gamma_{i}
\end{aligned}
$$

Hence $\zeta=\xi$. This completes the proof of 2.1 .

\section{Derivations}

Let $\Lambda$ be in $\operatorname{Alg}(\mathcal{D})$ and let $M$ be a $\Lambda$ - $\Lambda^{\mathrm{op}}$ bimodule. This means that there are morphisms $p_{M}: \Lambda \otimes M \longrightarrow M$ and $q_{M}: M \otimes \Lambda \longrightarrow M$ satisfying the rules for right and left modules; and that $p_{M}\left(1 \otimes q_{M}\right)=q_{M}\left(p_{M} \otimes 1\right)$.

3.1. Definition. A derivation of $\Lambda$ to $M$ is a morphism $d: \Lambda \longrightarrow M$ such that $d p_{\Lambda}=$ $p_{M}(1 \otimes d)+q_{M}(d \otimes 1): \Lambda \otimes \Lambda \longrightarrow M$. It is clear that the set of derivations of $\Lambda$ to $M$ is a subgroup of $\operatorname{Hom}_{\mathcal{D}}(\Lambda, M)$. We denote this abelian group by $\operatorname{Der}(\Lambda, M)$.

3.2. Proposition. Let $C \Lambda=\left\{C_{n} \Lambda\right\}, n \geq 0$, be the complex in $\mathcal{D}$ such that $C_{n} \Lambda=$ $\Lambda^{(n+2)}$, and boundary operator $\delta=\sum(-1)^{i}\left(1 \otimes 1 \otimes \cdots \otimes p_{\Lambda} \otimes 1 \otimes \cdots \otimes 1\right)$. Then $C$ is an acyclic complex over $\Lambda$, i.e.

$$
\cdots \longrightarrow C_{n} \longrightarrow \cdots \stackrel{p_{\Lambda}}{\longrightarrow} C_{0}
$$

is exact.

Proof. For let $s_{n}: C_{n} \Lambda \longrightarrow C_{n+1} \Lambda$ by $s_{n}=\left((u \otimes 1) e_{\Lambda}^{-1}\right) \otimes 1 \otimes \cdots \otimes 1$ for $n \geq 0$, and $s_{-1}: \Lambda \longrightarrow C_{0}$ by $(u \otimes 1) e_{\Lambda}^{-1}$. Then in the usual way it is easily shown that $p_{\Lambda} s_{-1}=1$ and $s_{n-1} \delta_{n}+\delta_{n+1} s_{n}=1$ for $n \geq 0$.

3.3. Definition. $J \Lambda=\operatorname{ker} p_{\Lambda} \cong \operatorname{coker} \delta_{2}$. Since by [Mac Lane 1965, p. 81], the category of $\Lambda$-bimodules is abelian, $J \Lambda$ is a $\Lambda$-bimodule as well. If $\psi: \Lambda^{(3)} \longrightarrow J \Lambda$ is the cokernel of $\delta_{2}$, then $\operatorname{Hom}(\psi,-): \operatorname{Hom}_{\Lambda^{e}}(J \Lambda,-) \longrightarrow \operatorname{Hom}_{\Lambda^{e}}\left(\Lambda^{(3)},-\right)$ is a monomorphism onto the subgroup of that latter consisting of those maps whose composition with $\delta_{2}$ is 0 . We can map

$$
\phi: \operatorname{Der}(\Lambda,-) \longrightarrow \operatorname{Hom}_{\Lambda^{e}}\left(\Lambda^{(3)},-\right)
$$

by restriction of the isomorphism $\operatorname{Hom}_{\mathcal{D}}(\Lambda,-) \cong \operatorname{Hom}_{\Lambda^{e}}\left(\Lambda^{(3)},-\right)$, whence $\phi$ is a monomorphism also.

3.4. Proposition. $\operatorname{Im} \phi=\operatorname{Im} \operatorname{Hom}(\epsilon,-)$ and establishes a natural equivalence between $\operatorname{Der}(\Lambda,-)$ and $\operatorname{Hom}_{\Lambda^{e}}(J \Lambda,-)$. 
PROOF. After sorting through all the identifications made, it is easily seen that what I claim amounts to showing that $d: \Lambda \longrightarrow M$ is a derivation if and only if $p_{M}\left(1 \otimes q_{M}\right)(1 \otimes$ $d \otimes 1) \delta_{2}=0$. If we recall the defining identities of bimodules, it is a straightforward computation that

$$
\left.p_{M}\left(1 \otimes q_{M}\right)(1 \otimes d \otimes 1) \delta_{2}=p_{M}\left(1 \otimes q_{M}\right)\left(1 \otimes p_{M}\right)\left(1 \otimes d-d p_{\Lambda}+q_{M}(d \otimes 1)\right] \otimes 1\right)
$$

This gives one implication immediately. On the other hand, for any $f: \Lambda \longrightarrow M$,

$$
\begin{aligned}
p_{M}\left(1 \otimes q_{M}\right)(1 \otimes f \otimes 1)\left(u_{\Lambda} \otimes 1 \otimes u_{\Lambda}\right) & =p_{M}\left(1 \otimes q_{M}\right)\left(u_{\Lambda} \otimes f \otimes u_{\Lambda}\right)=p_{M}\left(u_{\Lambda} \otimes q_{M}\left(f \otimes u_{\Lambda}\right)\right) \\
& =p_{M}\left(u_{\Lambda} \otimes q_{M}\left(1 \otimes u_{\Lambda}\right)(f \otimes 1)\right)=p_{M}\left(u_{\Lambda} \otimes e_{M}(f \otimes 1)\right) \\
& =p_{M}\left(u_{\Lambda} \otimes f e_{\Lambda}\right)=p_{M}\left(u_{\Lambda} \otimes 1\right)\left(1 \otimes f e_{\Lambda}\right) \\
& =e_{M}\left(1 \otimes f e_{\Lambda}\right)=f e_{\Lambda} e_{\Lambda \otimes K}
\end{aligned}
$$

Since these $e$ are isomorphisms, the other implication follows.

3.5. Definition. Let $M$ be a $\Lambda$-bimodule as before. We let $M^{+}$denote the algebra whose underlying $\mathcal{D}$ object is $\Lambda+M$ and whose multiplication is that map from

$$
(\Lambda+M) \otimes(\Lambda+M) \cong \Lambda \otimes \Lambda+\Lambda \otimes M+M \otimes \Lambda
$$

whose matrix is

$$
\left\|\begin{array}{cccc}
p_{\Lambda} & 0 & 0 & 0 \\
0 & p_{M} & q_{M} & 0
\end{array}\right\|
$$

Let $\pi_{1}: M^{+} \longrightarrow \Lambda$ and $\pi_{2}: M^{+} \longrightarrow M$ be the coordinate projections. It is easily seen that $\pi_{1}$ is a surjection of algebras.

3.6. Proposition. The mapping $f \longrightarrow \pi_{2} f$ establishes an equivalence between $\{f \in$ $\left.\operatorname{Hom}_{\mathrm{Alg}(\mathcal{D})}\left(\Lambda, M^{+}\right) \mid \pi_{1} f=1\right\}$ and $\operatorname{Der}(\Lambda, M)$.

ProOF. Let $f$ be such a mapping. Then $f$ has a matrix $\left\|f_{1} \quad f_{2}\right\|$, where $f_{1}=\pi_{1} f=1$ and $f_{2}=\pi_{2} f$. Hence there is a one to one correspondence between such $f$ and maps $f_{2}: \Lambda \longrightarrow M$ such that $\left\|\begin{array}{c}1 \\ f_{2}\end{array}\right\|$ is an algebra homomorphism. This condition becomes

$$
\left\|\begin{array}{cccc}
p_{\Lambda} & 0 & 0 & 0 \\
0 & p_{M} & q_{M} & 0
\end{array}\right\|\left\|\begin{array}{c}
1 \otimes 1 \\
1 \otimes f_{2} \\
f_{2} \otimes 1 \\
f_{2} \otimes f_{2}
\end{array}\right\|=\left\|\begin{array}{c}
1 \\
f_{2}
\end{array}\right\| p_{\Lambda}
$$

which reduces to $p_{\Lambda}(1 \otimes 1)=p_{\Lambda}$, which is clear; and $p_{M}\left(1 \otimes f_{2}\right)+q_{M}\left(f_{2} \otimes 1\right)=f_{2} p_{\Lambda}$, which is the defining equation of a derivation. 
3.7. Definition. Let $(\mathcal{D}, \Lambda)$ denote the category whose objects are $\operatorname{Alg}(\mathcal{D})$ morphisms $\gamma: \Gamma \longrightarrow \Lambda$ and whose morphisms are commutative triangles

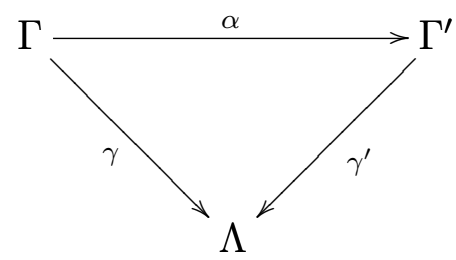

where $\alpha$ is a morphism in $\operatorname{Alg}(\mathcal{D})$. We may always consider $\pi_{1}: M^{+} \longrightarrow \Lambda$ as such an object. In that case, Proposition 3.6 becomes

3.8. Proposition. If $M^{+} \longrightarrow \Lambda$ is as above, then

$$
\operatorname{Hom}_{(\operatorname{Alg}(D), \Lambda)}\left(1_{\Lambda}, \pi_{1}\right) \cong \operatorname{Der}(\Lambda, M)
$$

\section{Modules}

Suppose that $\gamma: \Gamma \longrightarrow \Lambda$ is in $\operatorname{Alg}(\mathcal{D})$. We define functors

$$
\begin{gathered}
P=P_{\gamma}: \Lambda-\operatorname{Mod}(\mathcal{D}) \longrightarrow \Gamma-\operatorname{Mod}(\mathcal{D}) \\
Q=Q_{\gamma}: \Gamma-\operatorname{Mod}(\mathcal{D}) \longrightarrow \Lambda-\operatorname{Mod}(\mathcal{D})
\end{gathered}
$$

by setting, for a $\Lambda$-module $\left(M, p_{M}\right)$ and a $\Gamma$-module $\left(N, p_{N}\right), P\left(M, p_{M}\right)=\left(M, p_{M} \otimes 1\right)$ and $Q\left(N, p_{N}\right)=\left(P^{\mathrm{op}}(\Lambda) \otimes_{\Gamma} N, P^{\mathrm{op}}\left(p_{\Lambda}\right) \otimes_{\Gamma} p_{N}\right)$, where $P^{\mathrm{op}}$ is the functor analogous to $P$ for right $\Lambda$-modules.

\subsection{Theorem. $Q$ is left adjoint to $P$.}

Proof. First we define a morphism $\eta: 1 \longrightarrow P Q$. By abuse of notation, $\eta_{N}: N \longrightarrow A \otimes_{\Gamma} N$ is defined by te $t\left(u_{\Lambda} \otimes 1\right) e_{N}^{-1}$ where $t: \Lambda \otimes N \longrightarrow \Lambda \otimes_{\Gamma} N$ is as defined in Mac Lane 1965, p. 82]. We must show that

$$
\eta_{N} p_{N}=p_{P Q(N)}\left(1 \otimes \eta_{N}\right): \Gamma \otimes N \longrightarrow \Lambda \otimes_{\Gamma} N
$$


In the diagram

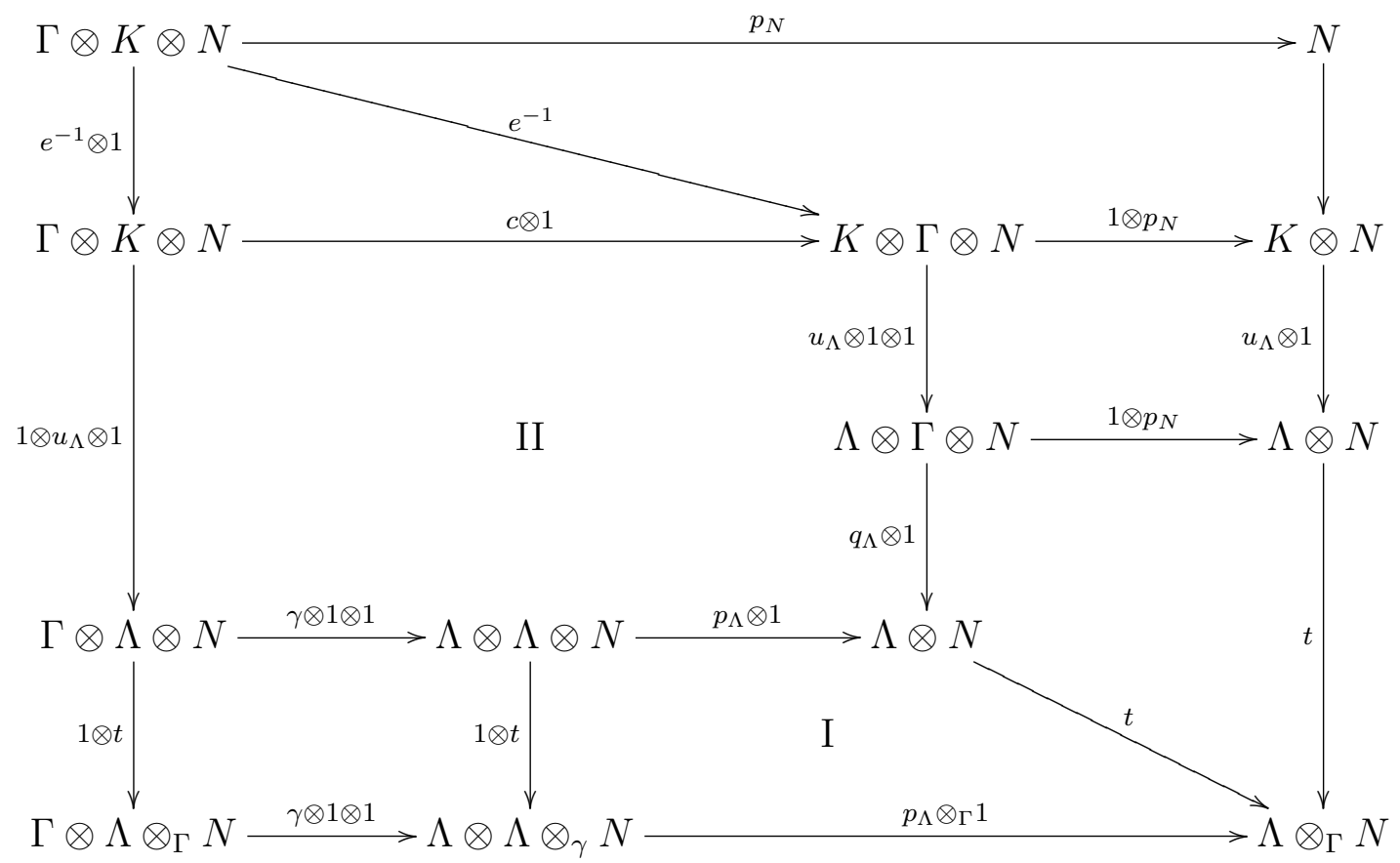

every subdiagram except those labeled I and II commutes either by a coherence or naturality. The equation $q_{\Lambda}=p_{\Lambda}(1 \otimes \gamma): \Lambda \otimes \Gamma \longrightarrow \Lambda$ is just the operation of $\Gamma$ on $\Lambda$ and I commutes by definition of $\otimes_{\Gamma}$.

As for II, in the diagram

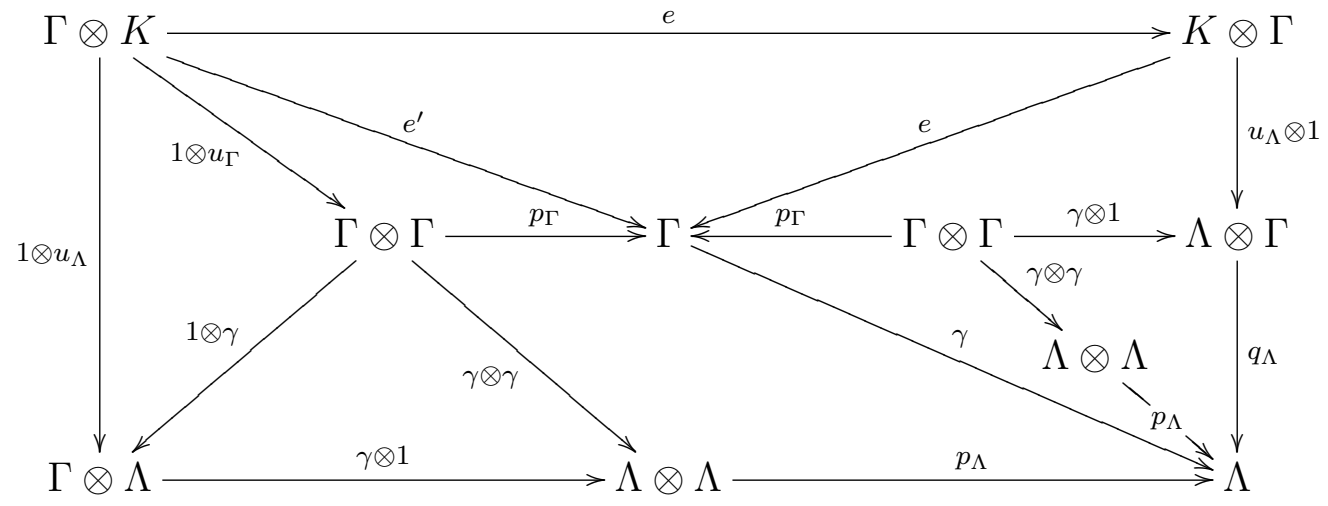

every subdiagram is commutative either because if coherence, naturality, or because $\gamma$ is a morphism of algebras. Tensoring the outer square with $N$ gives the desired result. To complete the proof we must show that, given a $\Gamma$-linear map $\alpha: N \longrightarrow P M$, we can find a unique $\Lambda$-linear map $\beta: Q N \longrightarrow M$ such that $P \beta \cdot \eta_{N}=\alpha$. Since $\Lambda \otimes_{\Gamma} N$ us the cokernel 
of $q_{\Lambda} \otimes 1-1 \otimes p_{N}: \Lambda \otimes \Gamma \otimes N \longrightarrow \Lambda \otimes N$, we have

$$
\begin{aligned}
p_{M}(1 \otimes \alpha)\left(q_{\Lambda} \otimes 1\right) & =p_{M}\left(p_{\Lambda} \otimes 1\right)(1 \otimes \gamma \otimes 1)(1 \otimes 1 \otimes \alpha) \\
& =p_{M}\left(1 \otimes p_{M}\right)(1 \otimes \gamma \otimes 1)(1 \otimes 1 \otimes \alpha) \\
& =p_{M}\left(1 \otimes p_{M}(\gamma \otimes 1)(1 \otimes \alpha)\right) \\
& =p_{M}\left(1 \otimes \alpha p_{M}\right)=p_{M}(1 \otimes \alpha)\left(1 \otimes p_{N}\right)
\end{aligned}
$$

Hence $p_{M}(1 \otimes \alpha)$ induces a map $\beta: \Lambda \otimes_{\Gamma} N \rightarrow M$. To show this is $\Lambda$-linear we must show that $p_{M}(1 \otimes \beta)=\beta\left(p_{\Lambda} \otimes_{\Gamma} 1\right)$. But since these are cokernels it reduces to showing that

$$
\begin{aligned}
{\left[1 \otimes p_{M}(1 \otimes \alpha)\right] } & =p_{M}\left(1 \otimes p_{M}\right)(1 \otimes 1 \otimes \alpha) \\
& =p_{M}\left(p_{\Lambda} \otimes 1\right)(1 \otimes 1 \otimes \alpha)=p_{M}(1 \otimes \alpha)\left(p_{\Lambda} \otimes 1\right)
\end{aligned}
$$

The fact that $P \beta \cdot \eta_{N}=\alpha$ follows from the commutativity of

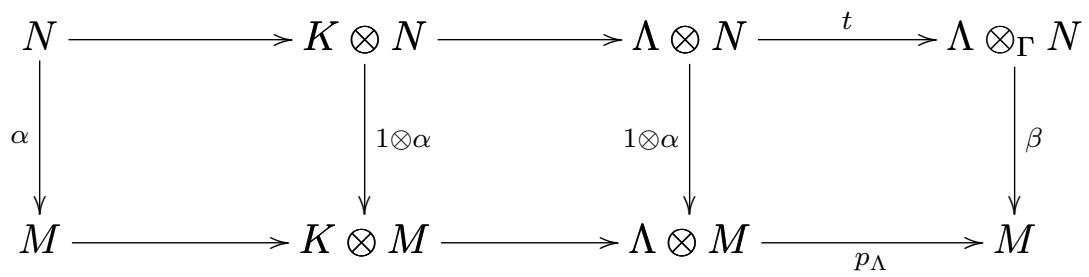

together with the fact that the bottom row is just the identity. Now suppose that $\beta^{\prime}$ : $\Lambda \otimes_{\Gamma} N \longrightarrow M$ is another $\Lambda$-linear map with $P \beta^{\prime} \cdot \eta_{N}=\alpha$. Then $\beta^{\prime} t: \Lambda: \Lambda \otimes N \longrightarrow M$ is a $\Lambda$-linear map such that $\alpha=\beta^{\prime} t\left(u_{\Lambda} \otimes 1\right) e_{N}^{-1}$. Since $\beta^{\prime} t$ is $\Lambda$-linear, $\beta^{\prime} t\left(p_{\Lambda} \otimes 1\right)=p_{M}\left(1 \otimes \beta^{\prime} t\right)$. From the former, we get

$$
1 \otimes \alpha=\left(1 \otimes \beta^{\prime} t\right)\left(1 \otimes u_{\Lambda} \otimes 1\right)\left(1 \otimes e_{N}^{-1}\right)
$$

and then

$$
\begin{aligned}
p_{M}(1 \otimes \alpha) & =p_{M}\left(1 \otimes \beta^{\prime} t\right)\left(1 \otimes u_{\Lambda} \otimes 1\right)\left(1 \otimes e_{N}^{-1}\right) \\
& =\beta^{\prime} t\left(p_{\Lambda} \otimes 1\right)\left(1 \otimes u_{\Lambda} \otimes e_{N}^{-1}\right) \\
& =\beta^{\prime} t\left(e_{\Lambda}^{\prime} \otimes e_{N}^{-1}\right)
\end{aligned}
$$

But by coherence, $e_{\Lambda}^{\prime} \otimes e_{N}^{-1}: \Lambda \otimes N \longrightarrow \Lambda \otimes K \otimes N \rightarrow \Lambda \otimes N$ must be the identity, so $\beta^{\prime} t=p_{M}(1 \otimes a l p h a)=\beta t$. Since $t$ is an epimorphism, the result follows.

Henceforth, we will not distinguish between $M$ and $P(M)$. We have already noted that if $M$ is a $\Lambda$-module, then

$$
\operatorname{Hom}_{\operatorname{Alg}(D), \Lambda}\left(I_{\Lambda}, \pi_{1}\right) \cong \operatorname{Der}(\Lambda, M)
$$

Now if $\gamma: \Gamma \longrightarrow \Lambda$ is in $(\operatorname{Alg}(\mathcal{D}), \Lambda)$, the fact that

$$
\operatorname{Der}(\Gamma, M) \cong \operatorname{Hom}_{(\operatorname{Alg}(\mathcal{D}), \Lambda)}
$$

can be proved in exactly the same way. Thus I have shown half of the following: 
4.2. Theorem. $\pi_{1}: M^{+} \longrightarrow \Lambda$ is an abelian object of $(\operatorname{Alg}(\mathcal{D}), \Lambda)$. If $\gamma: \Gamma \rightarrow \Lambda$ is an abelian object of that category and $N=\operatorname{ker} \gamma$, then $N=P(M)$ for suitable $M$ and $\gamma \cong \pi_{1}$.

Proof. If $\gamma: \Gamma \longrightarrow \Lambda$ is an abelian object, then $\operatorname{Hom}(1, \gamma)$ is also an abelian group. If $i$ denotes its identity, then $i: \Gamma \longrightarrow \Lambda$ is a right inverse to $\gamma$ so that $\gamma$ is an epimorphism. It is easily seen that $\operatorname{ker} \gamma=N$ is a $\Gamma$-bimodule (for $\gamma: \Gamma \longrightarrow \Lambda$ is also a morphism of of $\Gamma$-bimodules) and so $M=P_{i}(N)$ is a $\Lambda$-module. Essentially, $i$ induces a $\Lambda$-structure on $N$. Now, considered as objects of $\mathcal{D}, \gamma$ has a right inverse so that $\Gamma \cong \Lambda+M$ and $p_{\Gamma}$ has a representation as a matrix

$$
\left\|\begin{array}{cccc}
\alpha_{11} & \alpha_{12} & \alpha_{13} & \alpha_{14} \\
\alpha_{21} & \alpha_{22} & \alpha_{23} & \alpha_{24}
\end{array}\right\|
$$

with respect to the basis indicated in the expansion

$$
(\Lambda+M) \otimes(\Lambda+M)=\Lambda \otimes \Lambda+\Lambda \otimes M+M \otimes \Lambda+M \otimes M
$$

The fact that with repect to these bases, $i$ has matrix $\left\|\begin{array}{l}1 \\ 0\end{array}\right\|$ and is a morphism in $(\operatorname{Alg}(\mathcal{D}), \Lambda)$ shows that $\alpha_{21}=0$ and is in $(\operatorname{Alg}(\mathcal{D}), \Lambda)$, a similar computation shows that $\alpha_{12}=\alpha_{13}=\alpha_{14}=0$. We will be finished if we show that $\alpha=\alpha_{24}=0$. Since $\gamma: \Gamma \longrightarrow \Lambda$ is an abelian object there is a map of $\gamma \times \gamma \rightarrow \gamma$ where the product is in $(\operatorname{Alg}(\mathcal{D}), \Lambda)$. It is easily checked that $\gamma \times \gamma$ has the underlying object $\Sigma=\Lambda+M+M^{\prime}$ where $M=M^{\prime}$ is given a different name for the purpose of keeping track of an ordered basis. This algebra has multiplication map

$$
\begin{aligned}
p_{\Sigma}: \Sigma \otimes \Sigma & \cong \Lambda \otimes \Lambda+\Lambda \otimes M+\Lambda \otimes M^{\prime}+M \otimes \Lambda+M \otimes M \\
& +M \otimes M^{\prime}+M^{\prime} \otimes \Lambda+M^{\prime} \otimes M+M^{\prime} \otimes M^{\prime} \\
& \longrightarrow \Sigma
\end{aligned}
$$

whose matrix is

$$
\left\|\begin{array}{ccccccccc}
p_{\Lambda} & 0 & 0 & 0 & 0 & 0 & 0 & 0 & 0 \\
0 & p_{M} & 0 & q_{M} & \alpha & 0 & 0 & 0 & 0 \\
0 & 0 & p_{M} & 0 & 0 & 0 & q_{M} & 0 & \alpha
\end{array}\right\|
$$

If $i_{1}: \Gamma \longrightarrow \Sigma$ and $i_{2}: \Gamma \longrightarrow \Sigma$ are the injections (with matrices

$$
\left\|\begin{array}{ll}
1 & 0 \\
0 & 1 \\
0 & 0
\end{array}\right\| \quad \text { and } \quad\left\|\begin{array}{ll}
1 & 0 \\
0 & 0 \\
0 & 1
\end{array}\right\|
$$

respectively), then a group law on $\Gamma$ would be a map $\theta: \Sigma \longrightarrow \Gamma$ in $(\operatorname{Alg}(\mathcal{D}), \Lambda)$ satisfying (among other conditions) $\theta i_{1}=\theta i_{2}=1$. Writing out $\theta$ in matrix form, these conditions imply that $\alpha=0$. 
4.3. REMARK. The meaning of this theorem is that the categories of $\Lambda$-modules for the $\mathcal{D}$-algebra $\Lambda$, [Mac Lane 1965, p. 81], and $\Lambda$-modules for the $\mathbf{T}$-algebras $\Lambda$ Barr \& Beck 1966, Beck 1967] are isomorphic.

\section{Cohomology}

5.1. Theorem. If $\gamma: \Gamma \longrightarrow \Lambda$ is in $(\operatorname{Alg}(\mathcal{D}), \Lambda), \phi=\gamma \otimes \gamma^{\mathrm{op}}: \Gamma^{e} \longrightarrow \Lambda^{e}$ and $M^{+}$is an abelian object in that category, then there is an isomorphism

$$
H^{n}\left(\Gamma, M^{+}\right)_{\Lambda} \longrightarrow \begin{cases}\operatorname{Der}\left(\Gamma, P_{\phi}(M)\right), & \text { if } n=0 \\ H^{n+1}\left(\Gamma, P_{\phi}(M)\right), & \text { if } n>0\end{cases}
$$

where the left hand side refers to the triple cohomology and the right hand side is the cohomology of Mac Lane's bar construction.

Proof. We use the method of acyclic models as explained in detail in [Barr \& Beck 1966]. We define standard cochain complexes $L, S:(\operatorname{Alg}(\mathcal{D}), \Lambda) \longrightarrow \mathcal{A} b$, the category of abelian groups. Let $L_{n}(\Gamma, M)=\operatorname{Hom}_{\Gamma^{e}}\left(\Gamma^{n+3}, M\right)$, where again, we are letting $M$ stand for some $P(M)$ and

$$
S^{n}(\Gamma, M)=\operatorname{Hom}_{(\operatorname{Alg}(\mathcal{D}), \Lambda)}\left(G \Gamma^{n+1}, M^{+}\right)
$$

The boundary operator in $L$ takes $f$ to

$$
p_{M}(1 \otimes f)+\sum(-1)^{i}\left(1 \otimes \cdots \otimes p_{\Gamma} \otimes \cdots \otimes 1\right)+(-1)^{n+1} q_{M}(f \otimes 1)
$$

where $p_{\Gamma}$ is its multiplication map and $p_{M}$ and $q_{M}$ are the bimodule operations on $M$. The coboundary in $S$ takes $f$ to $\sum(-1)^{i} \operatorname{Hom}\left(f \cdot G^{i} \epsilon G^{n-i}, M\right)$. Then it is clear that $L$ and $S$ are standard complexes for the modified Mac Lane cohomology appearing on the right of Theorem 4.1 and the triple cohomomogy, respectively. Using $\eta$ we may show, exactly as in [Barr \& Beck 1966], that $S$ is $G$-representable and $G$-acyclic on models. If we can show the same for $L$ and that $L^{-1} \cong S^{-1}$, we will be through. If $\zeta=\eta \Gamma: \Gamma \longrightarrow G \Gamma$ is the adjointness morphism (in $\mathcal{D}$ ), it induces a map $\zeta^{(n+1)}: \Gamma^{(n+1)} \longrightarrow G \Gamma^{(n+1)}$. We have

$$
\begin{aligned}
L_{n}(\Gamma, M) & =\operatorname{Hom}_{\Gamma^{e}}\left(\Gamma^{(n+3)}, M\right) \cong \operatorname{Hom}\left(\Gamma^{(n+1)}, M\right) \\
& \stackrel{\operatorname{Hom}\left(\zeta^{n+1}, M\right)}{\longrightarrow} \operatorname{Hom}\left(G \Gamma^{(n+1)}, M\right) \cong \operatorname{Hom}_{G \Gamma^{e}}\left(G \Gamma^{(n+3)}, M\right) \\
& =L^{n}(G \Gamma, M)
\end{aligned}
$$

a $G$-representation of $L$. To show that $L$ is $G$-acyclic, we choose $f \in L^{n}(G \Gamma, M)$ and define $s f \in L^{n-1}(G \Gamma, M)$ as follows. Let

$$
\alpha\left(i_{0}, \ldots, i_{n+1}\right): \Gamma^{\left(i_{0}\right)} \otimes \ldots \otimes \Gamma^{\left(i_{n+1}\right)} \longrightarrow G \Gamma^{(n+2)}
$$

Denote the inclusion and define $s f$ by

$$
s f \cdot \alpha\left(i_{0}, 0, i_{n}, \ldots, i_{n+1}\right)=f \cdot \alpha\left(i_{0}, 0,0, i_{n}, \ldots, i_{n+1}\right) \times\left(1 \otimes e_{K}^{-1} 1 \otimes \cdots \otimes 1\right)
$$


for $i_{1}=0$ and inductively, for $i_{1}>0$

$$
\begin{aligned}
& s f \cdot \alpha\left(i_{0}, i_{1}, \ldots, i_{n+1}\right) \\
& =s f \cdot \alpha\left(i_{0}+1, i_{1}-1, i_{2}, \ldots, i_{n}\right)\left[\left(\sigma\left(i_{0}, 1\right) \otimes 1\right)\left(1 \otimes \sigma\left(1, i_{1}-1\right)^{-1}\right) \otimes 1 \otimes \cdots \otimes 1\right] \\
& -f \cdot \alpha\left(i_{0}, 1, i_{1}-1, i_{2}, \ldots, i_{n}\right)\left(1 \otimes \sigma\left(1, i_{1}-1\right)^{-1} \otimes 1 \otimes \cdots \otimes 1\right)
\end{aligned}
$$

Then, just as in Barr \& Beck 1966, $s$ may be shown to be a contraction in $L(G \Gamma, M)$.

$$
L^{-1}(\Gamma, M) \cong \operatorname{Hom}_{\Gamma^{e}}(J \Gamma, M) \cong \operatorname{Der}(\Gamma, M) \cong \operatorname{Hom}_{(\operatorname{Alg}(\mathcal{D}), \Lambda)}\left(\Gamma, M^{+}\right)
$$

by the results of Section 3, and it is shown in [Beck 1967] that the latter is

$$
H^{0}(S(\gamma, M)) \cong S^{-1}(\Gamma, M)
$$

. This completes the proof.

\section{References}

M. Barr, J. Beck (1966), Acyclic models and triples. COCA, 336-343.

J. Beck (1967), Triples, Algebras and Cohomology. Dissertation, Columbia University.

S. Mac Lane (1963), Homology. Springer.

S. Mac Lane (1965), Categorical algebra. Bull. Amer. Math. Soc. 71, 40-106 